periodic under $f$, then $x$ is also almost periodic under $f^{n}$ for every integer $n$.

The proof proceeds easily from Lemma 1, Remark 2, and Theorems 5 and 6.

\title{
BIBLIOGRAPHY
}

1. G. D. Birkhoff, Dynamical systems, Amer. Math. Soc. Colloquium Publications, vol. 9, New York, 1927.

2. G. D. Birkhoff and P. A. Smith, Structure analysis of surface transformations, J. Math. Pures Appl. vol. 7 (1928) pp. 345-379.

3. D. W. Hall and J. L. Kelley, Periodic types of transformations, Duke Math. J. vol. 8 (1941) pp. 625-630.

4. J. L. Kelley, A decomposition of compact continua and related theorems on fixed sets under continuous transformations, Proc. Nat. Acad. Sci. U.S.A. vol. 26 (1940) pp. 192-194.

5. G. T. Whyburn, Analytic topology, Amer. Math. Soc. Colloquium Publications, vol. 28, New York, 1942.

UNIVERSITY OF VIRGINIA

\section{SOME PROPERTIES OF SUMMABILITY. II ${ }^{1}$}

\section{J. D. HILL}

1. Summability of bounded sequences. It follows from a well known result of $H$. Steinhaus ${ }^{2}$ that no regular matrix method of summability can be effective for (that is, assign a finite limit to) every element in the space $(m)$ of bounded sequences. The object of this note is to consider some questions suggested by this fact. The first of these may be formulated as follows. If $A$ is a given regular matrix method let $J_{A}$ denote the set of all $A$-summable bounded sequences. We then ask what are necessary and sufficient conditions on a subset $E$ of $(m)$ in order that there exist a regular $A$ such that $E \subset J_{A}$ ? In Theorem 1 below it is shown that the separability of $E$ is a sufficient condition. It seems unlikely that this condition is necessary although we have been unable to decide the question. It is clearly equivalent to the question of whether every $J_{A}$ is separable.

TheOREM 1. Let $E$ be an arbitrary separable subset of $(m)$. Then every regular matrix $A=\left(a_{m k}\right)$ contains a (necessarily regular) row-submatrix $B=\left(a_{m_{i} k}\right)$ such that $E \subset J_{B}$.

Received by the editors November 6, 1943.

1 This note is in the nature of an appendix to the paper cited in footnote 4.

$2 \mathrm{H}$. Steinhaus, Some remarks on the generalizations of the notion of limit (in Polish), Prace Matematyczno-Fizyczne vol. 22 (1921) pp. 121-134. See also I. Schur, Über lineare Transformationen in der Theorie der unendlichen Reihen, J. Reine Angew. Math. vol. 151 (1921) pp. 79-111. 
Proof. The proof depends on an application of the so-called diagonal process. Let $D$ denote a dense denumerable subset of $E$ of points $x_{n} \equiv\left\{s_{k}^{n}\right\}$ for $n=1,2,3, \cdots$, and let $A=\left(a_{m k}\right)$ be an arbitrary regular matrix. By the Silverman-Toeplitz conditions the sequence $\left\{t_{m}^{1} \equiv \sum_{k} a_{m k} s_{k}^{1}\right\}$ is bounded and therefore contains a convergent subsequence $\left\{t_{m_{i}}^{1}\right\}$, where we may suppose that $m_{1}=1$. Let $a_{m_{i} k} \equiv a_{i k}^{1}$ for all $i$ and $k$, and select a convergent subsequence $\left\{t_{i_{i}}^{2}\right\}$ from the bounded sequence $\left\{t_{i}^{2} \equiv \sum_{k} a_{i k}^{1} s_{k}^{2}\right\}$ so that $i_{1}=1$ and $i_{2}=2$. Next let $a_{i, k}^{1} \equiv a_{j k}^{2}$ for all $j$ and $k$, proceed to $\left\{s_{k}^{3}\right\}$, and so forth. We define in this way a sequence of regular matrices $\left(a_{m k}\right),\left(a_{m k}^{1}\right),\left(a_{m k}^{2}\right), \cdots$, $\left(a_{m \mathrm{k}}^{n}\right), \cdots$, each a row-submatrix of the preceding. The "diagonal matrix" $\left(a_{m k}^{m}\right) \equiv B$ is evidently regular and by its construction it is clearly effective for each $x_{n}$ in $D$. To show that $B$ is also effective in $E-D$ we first observe that the operation $y=B(x)$, where $B(x)$ $\equiv\left\{\sum_{k} a_{m k}^{m} s_{k}\right\}$ and $x \equiv\left\{s_{k}\right\}$, is linear on $(m)$ to $(m)$. Then if $x_{0}$ is any point of $E-D$ there exists a subsequence $\left\{x_{n_{i}}\right\}$ of $D$ that converges to $x_{0}$. Setting $y_{i} \equiv B\left(x_{n_{i}}\right)$ we have $y_{0} \equiv \lim _{i} y_{i}=\lim _{i} B\left(x_{n_{i}}\right)=B\left(x_{0}\right)$. Since $y_{i}$ belongs to $(c)$, a closed subset of $(m)$, it follows that $y_{0}$ belongs to (c). This completes the proof.

2. A property of reversible methods. Let us first recall the following definitions. The method $A$ corresponding to the matrix $\left(a_{m k}\right)$ is said to be reversible ${ }^{3}$ if the system of equations

$$
t_{m}=\sum_{k=1}^{\infty} a_{m k} s_{k} \quad(m=1,2,3, \cdots)
$$

has a unique solution $\left\{s_{k}\right\}$ corresponding to each convergent sequence $\left\{t_{m}\right\}$. The method $A$ is said to be of ${ }^{3}$ type $M$ if the conditions

$$
\sum_{m=1}^{\infty}\left|u_{m}\right|<\infty, \quad \sum_{m=1}^{\infty} u_{m} a_{m k}=0 \quad(k=1,2,3, \cdots)
$$

always imply $u_{m}=0(m=1,2,3, \cdots)$. Finally, every method $A$ which is simultaneously regular, reversible, and of type $M$, is said to be perfect ${ }^{3}$.

We now ask if every regular method of summability which is stronger than convergence is necessarily effective for some unbounded sequences. For the class of reversible matrix methods an affirmative answer is supplied by the following theorems. These theorems bring to light the fact that while perfect methods of summability may be equivalent to convergence, reversible regular methods not of type $M$ are always stronger than convergence.

${ }^{3} \mathrm{~S}$. Banach, Thêorie des opérations linéaires, Warsaw, 1932, p. 90. 
THEOREM 2. Every perfect method of summability which is stronger than convergence is effective for some unbounded sequences.

Proof. Let $A=\left(a_{m k}\right)$ be an arbitrary perfect matrix, stronger than convergence, and let $F_{A}$ denote the set of all $A$-summable sequences. Then the transformation (2.1) of $F_{A}$ into $(c)$ has an inverse of the form ${ }^{4}$

$$
s_{k}=\sum_{m=0}^{\infty} A_{k m} t_{m} \quad(k=1,2,3, \cdots),
$$

where $t_{0} \equiv \lim _{m} t_{m}$ and $\sum_{m=0}^{\infty}\left|A_{k m}\right|<\infty(k=1,2,3, \cdots)$. If we let $x \equiv\left\{s_{k}\right\}$ and $y \equiv\left\{t_{m}\right\}$ we may express (2.1) as $y=A(x)$ and (2.2) as $x=A^{-1}(y)$.

Since $A$ is of type $M$ it follows from a lemma of Banach $^{5}$ that as $x$ ranges over $(c)$ the set $G_{A}$ of all points $y=A(x)$ is dense in $(c)$.

To give an indirect proof we assume now that $F_{A} \subset(m)$, that is, that every $A$-summable sequence is bounded. As a consequence it follows easily that the operation $x=A^{-1}(y)$ is then linear on $(c)$ to $F_{A}$. Let $x_{0}$ be an arbitrary point of $F_{A}$ and set $y_{0}=A\left(x_{0}\right)$. Since $y_{0}$ is in $(c)$ there exists, by the foregoing remark, a sequence $\left\{y_{n}\right\}$ in $G_{A}$, where $y_{n}=A\left(x_{n}\right)$ for certain $x_{n}$ in $(c)$, such that $y_{n} \rightarrow y_{0}$. But then $x_{n}=A^{-1}\left(y_{n}\right)$ $\rightarrow A^{-1}\left(y_{0}\right)=x_{0}$, and since $(c)$ is complete we see that $x_{0}$ belongs to $(c)$. Thus $A$ is equivalent to convergence, and this contradiction completes the proof.

THEOREM 3. Every reversible regular method $A$ which is not of type $M$ is effective for some unbounded sequences.

Proof. As $x$ ranges over $(m)$ we denote by $H_{A}$ the set of all points $y=A(x)$. By an easy extension of the previously mentioned lemma of Banach, it has been shown ${ }^{6}$ that $A$ is of type $M$ if and only if the points $z_{n} \equiv\left\{\delta_{k}^{n}\right\}$ for $n=1,2,3, \cdots$ (where $\delta_{k}^{n}$ is the Kronecker symbol) are points of $\bar{H}_{A}$, the closure of $H_{A}$. Hence if $A$ is not of type $M$ at least one of the points $z_{n}$, say $z_{r}$, is not in $\bar{H}_{A}$. Since $A$ is reversible and $z_{r}$ belongs to $(c)$, the equation $A(x)=z_{r}$ has a unique solution $x=x_{r}$. It is clear that $x_{r}$ can not be a point of $(m)$ and this fact establishes the theorem.

\footnotetext{
4 See J. D. Hill, Some properties of summability, Duke Math. J. vol. 9 (1942) pp. 373-381; in particular, p. 376.

${ }^{5}$ See footnote 3, p. 93, Lemma 2.

- See J. D. Hill, On perfect methods of summability, Duke Math. J. vol. 3 (1937) pp. 702-714; in particular p. 704, Theorem 4.
} 
An alternative proof of this theorem may be given as follows. Under the assumed hypotheses on $A$ we conclude from known theorems that ${ }^{7}$ there exists a regular method $B$, not weaker than $A$, with which $A$ is inconsistent; and that ${ }^{8} A$ and $B$ are consistent for bounded sequences. Hence the inconsistency must occur for unbounded sequences.

3. Methods effective for no bounded divergent sequences. As our final question we ask if there exist regular methods, stronger than convergence, which are effective for no bounded divergent sequences. An example will suffice to show that the answer is affirmative.

Example. We define matrices $B=\left(b_{m k}\right)$ and $C=\left(c_{m k}\right)$ as follows: $b_{11} \equiv b_{k+1, k} \equiv 1 \quad(k=1,2,3, \cdots)$, and $b_{m k} \equiv 0$ otherwise; $c_{m m} \equiv 1 / m$ $(m=1,2,3, \cdots)$, and $c_{m k} \equiv 0$ otherwise. The matrix $B$ is a regular matrix that leaves invariant the limit points of each bounded sequence. The matrix $C$, on the other hand, assigns the limit 0 to every bounded sequence. Consequently, the sum matrix $A \equiv B+C$ leaves invariant the limit points of each bounded sequence, and is therefore effective for no bounded divergent sequence. One easily verifies, however, that $A$ assigns the limit 0 to the unbounded sequence $\left\{(-1)^{k-1} k !\right\}$.

The matrix $A$ of this example is a simple instance of a Raff matrix, ${ }^{9}$ namely, a matrix that leaves invariant the limit points of each bounded sequence. Every Raff matrix which is stronger than convergence will therefore possess the property in question, for example, every reversible Raff matrix which is not of type $M$ (see Theorem 3 above).

\section{Michigan State College}

7 See footnote 4, p. 380, Theorem 5 .

8 See footnote 3, p. 95, Theorème 11.

${ }^{\circ}$ Hermann Raff, Zur Theorie der lineare Transformationen, Math. Zeit. vol. 37 (1933) pp. 572-577. 